\title{
Systems of Delay Differential Equations: Analysis of a model with feedback
}

\author{
Carlos Héctor Daniel Alliera ${ }^{1}$ and Pablo Amster ${ }^{1,2}$ \\ ${ }^{1}$ Departamento de Matemática, FCEyN - Universidad de Buenos Aires, Pabellón I, Ciudad \\ Universitaria,Buenos Aires, Argentina.E-mails: pamster@dm.uba.ar calliera@dm.uba.ar \\ ${ }^{2} I M A S-C O N I C E T$
}

\begin{abstract}
Using topological degree theory, we prove the existence of positive periodic solutions of a system of delay differential equations for models with feedback arising on regulatory mechanisms in which selfregulation is relevant, e.g. in cell physiology. We study different models based on the cycle of testosterone and generalizations. The method in the present work allows to analyze and extend known results from a different perspective, shortening proofs and giving an alternative approach for the study of complex models.
\end{abstract}

Keywords: Systems of DDEs; Periodic solutions; Models with feedback.

\section{Introduction}

Self-regulatory models are common in nature, as described e.g. in $[8,11,13]$. To fix ideas, let us consider a system made up of a number of glands as a motivation. Each gland secretes a hormone that allows secretion in the next gland, which successively generates another hormone to stimulate the next one and so on. In the end, a final hormone is released which, by increasing its concentration, inhibits the secretion of previous hormones that allowed the production process. This generates the decay of the hormone to a minimum threshold that re-activates the cycle again. A similar behavior can be observed in other biochemical processes, such as enzymatic or bacterial models.

In many of these processes, it makes sense to consider the existence of delays in time. For example in [3], a model is proposed that establishes in relation to the hours of response between an increase in GnRH (Gonadotropin-releasing hormone) secretion and a pituitary FSH (Follicle-stimulating hormone) response under dietary treatment or minutes for responses to sociosexual signs as we can see in [7].

The existence of periodic solutions is an important issue in a large number of biological problems, e.g. population dynamics. There exists a wide literature providing different methods of analysis $[8,9,11,2]$. Here, we shall make use of the Brouwer topological degree in order to obtain sufficient conditions for the existence of periodic solutions in a model that generalizes a hormonal cycle. Specifically, our result arises on the fact that the feedback structure of the 
model induces, when the system is autonomous, the existence of a positive equilibrium. We shall prove that if the parameters of the model are not necessarily constant but periodic functions with a fixed period, then the system has a positive solution with the same period.

Brouwer's topological degree is a useful tool to find zeros of a continuous function $f$ defined over the closure of a bounded open subset of the $n$-dimensional space; in particular, it can be used to obtain equilibria in a wide variety of models with constant parameters. Its extension for compact perturbations of the identity, i. e. $f(x):=x-K(x)$ with $K$ compact, is due to Leray and Schauder and, as we shall see, it can be employed to prove the existence of periodic solutions of some of the mentioned models.

In this work, we study the existence of periodic solutions for a general model with feedback, namely the following system of delay differential equations:

$$
\left\{\begin{array}{l}
\frac{d x_{0}}{d t}=F\left(t, x_{n}\left(t-\tau_{0}\right)\right)-b_{0}\left(x_{0}(t)\right), \\
\frac{d x_{j}}{d t}=G_{j}\left(t, x_{j-1}\left(t-\varepsilon_{j}\right), x_{n}\left(t-\tau_{j}\right)\right)-b_{j}\left(x_{j}(t)\right), \quad 1 \leq j \leq n-1 . \\
\frac{d x_{n}}{d t}=H\left(t, x_{n-1}\left(t-\varepsilon_{n}\right)\right)-b_{n}\left(x_{n}(t)\right)
\end{array}\right.
$$

Here $\tau_{i}$ and $\varepsilon_{j}$, with $0 \leq i \leq n-1$ and $1 \leq j \leq n$ are fixed non negative delays in time.

Throughout this paper, it shall be assumed that the model satisfies the following features:

1. $F, H: \mathbb{R} \times[0,+\infty) \rightarrow[0, \infty)$ and $G_{j}: \mathbb{R}^{3} \rightarrow[0, \infty)$ with $j=1, \ldots, n-1$ are continuous and $\theta$-periodic in the first coordinate for some fixed period $\theta>0$.

2. $b_{i}:[0,+\infty) \rightarrow[0,+\infty)$ is a strictly increasing function with $b_{i}(0)=0$ for $i=0, \ldots, n$.

3. $F$ is nonincreasing in its second coordinate with $F(t, x)>0$ for all $x \geq 0$ and $\operatorname{Im}(F) \subseteq \operatorname{Im}\left(b_{0}\right)$.

4. $H$ is nondecreasing in its second coordinate with $H(t, x)>0$ for all $x>0$ and $\operatorname{Im}(H) \subseteq \operatorname{Im}\left(b_{n}\right)$.

5. $G_{j}$ is nondecreasing in its second coordinate and nonincreasing in its third coordinate, with $G_{j}(t, x, y)>0$ for $x>0$ and $\operatorname{Im}\left(G_{j}\right) \subseteq \operatorname{Im}\left(b_{j}\right)$, for $j=1, \ldots, n-1$.

Our main result reads:

Theorem 1. Assume that the previous conditions 1-5 hold. Then system (1) has at least one $\theta$-periodic solution $u=\left(x_{0}, x_{1}, \ldots, x_{n}\right)$ such that $x_{k}(t)>0$ for all $t$ and all $k$.

The paper is organized as follows. In the next section, we introduce a general setting of the abstract problem in the context of topological degree theory and give a proof of our main theorem. Moreover, we present an example based on a model of testosterone secretion in which the abstract result applies. In section 3, we present two alternative models for which our main theorem cannot be applied, so the method is adapted in order to prove the existence of periodic solutions. Finally, in the last section we summarize the results of this paper and discuss possible generalizations and future lines of research.

\section{Existence of positive periodic solutions}

We shall apply the continuation method in the positive cone

$$
\mathcal{K}:=\left\{u \in C_{\theta}: x_{0}, x_{1}, \ldots, x_{n} \geq 0\right\},
$$

where $C_{\theta}$ is the Banach space of continuous $\theta$ - periodic functions

$$
C_{\theta}:=\left\{u \in C\left(\mathbb{R}, \mathbb{R}^{n+1}\right): u(t)=u(t+\theta) \text { for all } t\right\}
$$

equipped with the standard uniform norm. Consider the linear operator $L: C^{1} \cap C_{\theta} \rightarrow C_{\theta}$ given by $\mathbf{L} u:=u^{\prime}$ and the nonlinear Nemiskii operator $\mathbf{N}: \mathcal{K} \rightarrow C_{\theta}$ defined as the right-hand side of system (1).

For convenience, the average of a $\theta$-periodic function $u$, namely $\bar{u}:=\frac{1}{T} \int_{0}^{T} u(t) d t$, shall be denoted by $\bar{u}$. Also, we shall employ the notation $u_{\max }$ and $u_{\min }$ to refer to its absolute maximum and minimum values respectively. Identifying $\mathbb{R}^{n+1}$ with the subset of constant functions of $C_{\theta}$, we may define the function $\phi:[0,+\infty)^{n+1} \rightarrow \mathbb{R}^{n+1}$ given by $\phi(x):=\overline{\mathbf{N} x}$, that is: 


$$
\phi\left(x_{0}, x_{1}, \ldots, x_{n}\right)=\left(\frac{1}{\theta} \int_{0}^{\theta} F\left(t, x_{n}\right) d t-b_{0}\left(x_{0}\right), \frac{1}{\theta} \int_{0}^{\theta} G_{1}\left(t, x_{1}, x_{n}\right) d t-b_{1}\left(x_{1}\right), \ldots, \frac{1}{\theta} \int_{0}^{\theta} H\left(t, x_{n-1}\right) d t-b_{n}\left(x_{n}\right)\right) .
$$

The following continuation theorem can be easily deduced from the standard topological degree methods (see e.g. [1]).

Theorem 2. Assume there exists $\Omega \subset \mathcal{K}^{\circ}$ open and bounded such that:

a) The equation $\mathbf{L} u=\lambda \mathbf{N} u$ has no solutions on $\partial \Omega$ for $0<\lambda<1$.

b) $\phi(u) \neq 0$ for all $u \in \partial \Omega \cap \mathbb{R}^{n+1}$.

c) $\operatorname{deg}\left(\phi, \Omega \cap \mathbb{R}^{n+1}, 0\right) \neq 0$, where 'deg' denotes the Brouwer degree.

Then (1) has at least one solution in $\bar{\Omega}$.

\section{Proof of Theorem 1}

In order to apply the continuation theorem, let us firstly obtain a priori bounds for the solutions of the system $\mathbf{L} u=\lambda \mathbf{N} u$ with $\lambda \in(0,1]$. These bounds will yield an appropriate choice of a subset $\Omega$ containing all possible solutions; thus, the degree computation below and Theorem 2 will imply that a solution effectively exists for $\lambda=1$. For convenience, we shall employ the notation $t^{*}$ (resp. $t_{*}$ ) to express the values in which an absolute maximum (resp. minimum) of each of the different functions is achieved.

To this end, let $u=\left(x_{0}, x_{1}, \ldots, x_{n}\right) \in \mathcal{K}$ be such a solution and suppose, in the first place, that $x_{0}$ achieves its absolute maximum $x_{0 \max }$ at some value $t^{*}$. Then $x_{0}^{\prime}\left(t^{*}\right)=0$ and hence

$$
b_{0}\left(x_{0 \max }\right)=F\left(t^{*}, x_{n}\left(t^{*}-\tau_{0}\right)\right) \leq F\left(t^{*}, 0\right) .
$$

Fixing a constant $\mathcal{M}_{0}>\max _{t \in \mathbb{R}} b_{0}^{-1}(F(t, 0))$, we conclude that $x_{0 \text { max }}<\mathcal{M}_{0}$. Next, denote again by $t^{*}$ the value for which the absolute maximum of $x_{1}$ is achieved, then

$$
b_{1}\left(x_{1 \max }\right)=G_{1}\left(t^{*}, x_{0}\left(t^{*}-\varepsilon_{1}\right), x_{n}\left(t^{*}-\tau_{1}\right)\right) \leq G_{1}\left(t^{*}, x_{0 \max }, 0\right) \leq G_{1}\left(t^{*}, \mathcal{M}_{0}, 0\right) .
$$

Thus, we may fix a constant $\mathcal{M}_{1}>\max _{t \in \mathbb{R}} b_{1}^{-1}\left(G_{1}\left(t, \mathcal{M}_{0}, 0\right)\right)$ and hence $x_{1 \max }<\mathcal{M}_{1}$. In the same way, for $j=$ $2, \ldots, n-1$ we fix constants $\mathcal{M}_{j}>\max _{t \in \mathbb{R}} b_{j}^{-1}\left(G_{j}\left(t, \mathcal{M}_{j-1}, 0\right)\right)$ so $x_{j}(t)<\mathcal{M}_{j}$ for all $t$. For the last equation, suppose that $x_{n}$ achieves its absolute maximum at some $t^{*}$, then

$$
b_{n}\left(x_{n \max }\right)=H\left(t^{*}, x_{n-1}\left(t^{*}-\varepsilon_{n}\right)\right) \leq H\left(t^{*}, \mathcal{M}_{n-1}\right) .
$$

Thus we may fix a constant $\mathcal{M}_{n}>\max _{t \in \mathbb{R}} b_{n}^{-1}\left(H\left(t, \mathcal{M}_{n-1}\right)\right)$ and conclude that $x_{n \text { max }}<\mathcal{M}_{n}$.

In order to obtain lower bounds, assume firstly that $x_{0}$ achieves its absolute minimum $x_{0 \text { min }}$ at some $t_{*}$, then as before $x_{0}^{\prime}\left(t_{*}\right)=0$, which implies:

$$
b_{0}\left(x_{0 \text { min }}\right)=F\left(t_{*}, x_{n}\left(t_{*}-\tau_{0}\right)\right) \geq F\left(t_{*}, \mathcal{M}_{n}\right)>0 .
$$

Then, choosing a positive constant $\mathfrak{m}_{0}<\min _{t \in \mathbb{R}} b_{0}^{-1}\left(F\left(t, \mathcal{M}_{0}\right)\right)$ it is seen that $x_{0 \text { min }}>\mathfrak{m}_{0}$. In the same way, we fix $\mathfrak{m}_{j}>0$ is such that $\mathfrak{m}_{j}<b_{j}^{-1}\left(G_{j}\left(t, \mathfrak{m}_{j-1}, \mathcal{M}_{n}\right)\right)$ for all $t$ and conclude that $x_{j}(t)>\mathfrak{m}_{j}$ for all $t$ and $1 \leq j \leq n-1$. Finally, fix a positive constant $\mathfrak{m}_{n}$ such that $\mathfrak{m}_{n}<b_{n}^{-1}\left(H\left(t, \mathfrak{m}_{n-1}\right)\right)$ for all $t$, then $x_{n}(t)>\mathfrak{m}_{n}$ for all $t$.

In other words, the first condition of the continuation theorem is satisfied over the set

$$
\Omega:=\left\{\left(x_{0}, x_{1}, \ldots, x_{n}\right) \in \mathcal{K}: \mathfrak{m}_{0}<x_{0}(t)<\mathcal{M}_{0}, \ldots, \mathfrak{m}_{j}<x_{j}(t)<\mathcal{M}_{j}, \ldots, \mathfrak{m}_{n}<x_{n}(t)<\mathcal{M}_{n} \text { for all } t, 1 \leq j \leq n-1\right\} .
$$

On the other hand, observe that $Q:=\Omega \cap \mathbb{R}^{n+1}=\left(\mathfrak{m}_{0}, \mathcal{M}_{0}\right) \times \ldots \times\left(\mathfrak{m}_{n}, \mathcal{M}_{n}\right)$, so we shall study the behaviour of the mapping $\phi$ over the faces of $Q$.

Let $x \in \bar{Q}$ and suppose $x_{0}=\mathfrak{m}_{0}$, then there exists $\hat{t}$ such that

$$
\phi_{0}(x)=\frac{1}{\theta} \int_{0}^{\theta} F\left(t, x_{n}\right) d t-b_{0}\left(\mathfrak{m}_{0}\right)=F\left(\hat{t}, x_{n}\right)-b_{0}\left(\mathfrak{m}_{0}\right)>F\left(\hat{t}, x_{n}\right)-F\left(\hat{t}, \mathcal{M}_{n}\right) \geq 0 .
$$

Similarly, if $x_{0}=\mathcal{M}_{0}$, then

$$
\phi_{0}(x)=\frac{1}{\theta} \int_{0}^{\theta} F\left(t, x_{n}\right) d t-b_{0}\left(\mathcal{M}_{0}\right)=F\left(\tilde{t}, x_{n}\right)-b_{0}\left(\mathcal{M}_{0}\right)<F\left(\tilde{t}, x_{n}\right)-F(\tilde{t}, 0) \leq 0 .
$$

In the same way, for all $j=1, . ., n-1$ if $x_{j}=\mathfrak{m}_{j}$ or $x_{j}=\mathcal{M}_{j}$ then

$$
\phi_{j}(x)=\frac{1}{\theta} \int_{0}^{\theta} G_{j}\left(t, x_{j-1}, x_{n}\right) d t-b_{j}\left(\mathfrak{m}_{j}\right)=G_{j}\left(\hat{t}, x_{j-1}, x_{n}\right)-b_{j}\left(\mathfrak{m}_{j}\right) \geq G\left(\hat{t}, \mathfrak{m}_{j-1}, \mathcal{M}_{n}\right)-b_{j}\left(\mathfrak{m}_{j}\right)>0
$$




$$
\phi_{j}(x)=\frac{1}{\theta} \int_{0}^{\theta} G_{j}\left(t, x_{j-1}, x_{n}\right) d t-b_{j}\left(\mathcal{M}_{j}\right)=G\left(\tilde{t}, x_{j-1}, x_{n}\right)-b_{j}\left(\mathcal{M}_{j}\right) \leq G\left(\tilde{t}, \mathcal{M}_{j-1}, 0\right)-b_{j}\left(\mathcal{M}_{j}\right)<0
$$

respectively. Finally,

$$
\phi_{n}\left(x_{0}, \ldots, x_{n-1}, \mathfrak{m}_{n}\right)=\frac{1}{\theta} \int_{0}^{\theta} H\left(t, x_{n-1}\right) d t-b_{n}\left(\mathfrak{m}_{n}\right)=H\left(\hat{t}, x_{n-1}\right)-b_{n}\left(\mathfrak{m}_{n}\right) \geq H\left(\hat{t}, \mathfrak{m}_{n-1}\right)-b_{n}\left(\mathfrak{m}_{n}\right)>0,
$$

and

$$
\phi_{n}\left(x_{0}, \ldots, x_{n-1}, \mathcal{M}_{n}\right)=\frac{1}{\theta} \int_{0}^{\theta} H\left(t, x_{n-1}\right) d t-b_{n}\left(\mathcal{M}_{n}\right)=H\left(\tilde{t}, x_{n-1}\right)-b_{n}\left(\mathcal{M}_{n}\right) \leq H\left(\tilde{t}, \mathcal{M}_{n-1}\right)-b_{n}\left(\mathcal{M}_{n}\right)<0 .
$$

We deduce that the second condition of the continuation theorem is fulfilled. Moreover, if we consider the homotopy $h: \overline{\mathrm{Q}} \times[0,1] \rightarrow \mathbb{R}^{n+1}$ given by

where

$$
h(x, \lambda):=(1-\lambda)(\mathfrak{p}-x)+\lambda \phi(x)
$$

$$
\mathfrak{p}:=\left(\frac{\mathcal{M}_{0}+\mathfrak{m}_{0}}{2}, \ldots, \frac{\mathcal{M}_{n}+\mathfrak{m}_{n}}{2}\right)
$$

then $h \neq 0$ on $\partial \mathrm{Q} \times[0,1]$. Indeed, if $h(x, \lambda)=0$ for some $x=\left(x_{0}, x_{1}, \ldots, x_{n}\right) \in \partial \mathrm{Q}$, then suppose for example that $x_{0}=\mathcal{M}_{0}$. Hence

$$
0=h_{0}(x, \lambda)=(1-\lambda) \overbrace{\frac{\mathfrak{m}_{0}-\mathcal{M}_{0}}{2}}^{<0}+\lambda \overbrace{\phi_{0}\left(\mathcal{M}_{0}, x_{1}, \ldots, x_{n}\right)}^{<0}<0,
$$

a contradiction. The other cases follow similarly. By the homotopy invariance of the Brouwer degree, we conclude that

$$
\operatorname{deg}(\phi, \mathrm{Q}, 0)=\operatorname{deg}(\mathfrak{p}-I d, \mathrm{Q}, 0)=(-1)^{n+1}
$$

and the proof is complete.

Remark 1. The previous proof does not depend on the choice of the delays; in particular, the result is also true for the non-delayed case. Observe also that, when the system is autonomous, the assumptions imply the existence of a positive equilibrium, which is a solution in $\mathcal{K}$. Thus, roughly speaking, the previous result shows that certain conditions, which imply the existence of a positive equilibrium for the autonomous case, also imply the existence of $\theta$-periodic solutions, provided that the constant parameters of the model are replaced by $\theta$-periodic functions. In the autonomous case, it is known that the presence of delays may cause the appearance of non-constant periodic solutions; thus, it is expected that extra solutions for the non-autonomous case might be obtained under appropriate relations between $\theta$ and the delays.

\subsection{Example: Model of Testosterone Secretion}

As a motivation, let us consider the following system described in [14] and [6], which is based on a model proposed by Smith [15] and motivated the general abstract model developed in this work.

Let us consider the model shown in Figure 2 for the cycle of the Testosterone hormone (see [13]), where the different variables denote the concentrations at time $t$ of the Luteinising Hormone $(L H)$, which is represented by $R(t)$, from hypothalamus, Luteinising Hormone Releasing Hormone $(L H R H)$, represented by $L(t)$, from Pituitary gland and Testosterone Hormone $(T H)$ from Testes in man, represented by $T(t)$.

A general autonomous model describing the biochemical interaction of the hormones $L H, L H R H$ and $T H$ in the male is presented.

The model structure consists of a negative feedback system of three delay differential equations. It is worthy to notice that high levels of $T$ affect the concentration of $R$ and $L$.

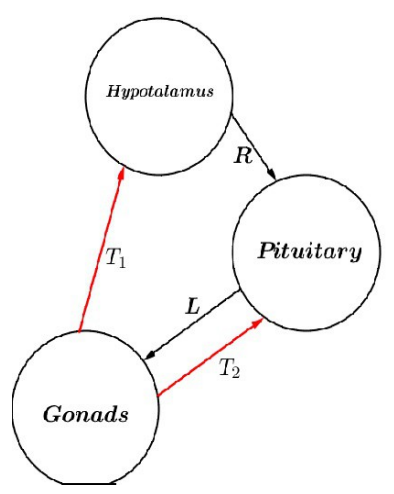

Figure 2: Hormone Testosterone cycle 


$$
\begin{aligned}
& \frac{d R}{d t}=F\left(t, T\left(t-\tau_{0}\right)\right)-b_{0}(R(t)), \\
& \frac{d L}{d t}=g_{1}\left(R\left(t-\tau_{1}\right), T\left(t-\tau_{2}\right)\right)-b_{1}(L(t)), \\
& \frac{d T}{d t}=g_{2}\left(L\left(t-\tau_{3}\right)\right)-b_{2}(T(t)) .
\end{aligned}
$$

This model has the form (1) and conditions 1-5 are satisfied if $b_{i}, F$ and $g_{j}$ are positive continuous functions for $i=0,1,2$ and $j=1,2$ with $g_{1}$ nondecreasing in its first variable and nonincreasing in the second one, $g_{2}$ nondecreasing, the delays $\tau_{i} \geq 0$ are constant and $F$ is $\theta$-periodic in $t$ and strictly decreasing in $T$ with

$$
F(t, 0) \leq \lim _{x \rightarrow+\infty} b_{0}(x), \quad \lim _{x \rightarrow+\infty} g_{i}(x) \leq \lim _{x \rightarrow+\infty} b_{i}(x) \quad i=1,2 .
$$

With this structure, Murray [13] proposed in 1989 a simpler non-delayed autonomous system, with:

$$
b_{i}(x)=\beta_{i} x, \quad \beta_{i}>0, \quad g_{1}(x, y)=\alpha_{1} x, \quad g_{2}(x)=\alpha_{2} x, \quad \alpha_{j}>0, \quad F(x)=\frac{\kappa_{1}}{\kappa_{2}+x^{m}}, m \in \mathbb{N}, \quad \tau_{i}=0
$$

where $\kappa_{j}>0$ are constants. The functions $g_{j}$ are related to the rates for productions of $L$ and $T$ and $b_{i}$ are the respective decay rates in the blood stream. It is assumed that each of these hormones is cleared from blood stream according to first order kinetics (see [6]). Here, the existence of a (unique) positive equilibrium is trivially proved. Our result implies that if $\kappa_{1}$ and $\kappa_{2}$ are replaced by $\theta$-periodic positive functions, then at least one $\theta$-periodic solution necessarily exists for arbitrary positive delays.

\section{Alternative models}

In what follows, we shall analyse non-autonomous versions of two models proposed by Greenhalgh [10] and Liu [12] respectively. Slight differences with respect to the model presented in the previous section imply that our main existence result is not directly applicable; however, we will find suitable conditions in order to prove the existence of $\theta$-periodic solutions.

\subsection{A new concentration: Leydig cells}

In 1990, Liu and Deng [12] proposed the following 4-dimensional model taking into account the number of interstitial Leydig cells, which produce testosterone in the presence of luteinizing hormone:

$$
\begin{aligned}
& \frac{d R}{d t}=\frac{a_{0}+a_{1} R(t)+a_{2} R(t)^{2}}{1+b_{1} T(t)+b_{2} T(t)^{2}+c_{1} R(t)+c_{2} R(t)^{2}}-d_{0} R(t), \\
& \frac{d L}{d t}=\frac{a_{3}+a_{4} R(t)}{1+b_{3} T(t)+c_{3} R(t)}-d_{1} L(t), \\
& \frac{d T}{d t}=k_{1}+k_{2} L(t)+\frac{k_{3}+k_{4} L(t)}{1+k_{5} L(t)} G(t)-d_{2} T(t), \\
& \frac{d G}{d t}=\frac{k_{6} L(t)}{1+k_{7} L(t)+q R(t) T(t)}-d_{3} G(t) .
\end{aligned}
$$

Here, $G$ is the number of effective Leydig cells. Because of its small rate of proliferation, $G$ is considered in [12] as a quasi-steady state so the system is reduced to a 3-dimensional system, although this assumption shall not be made in the present work. According to [12], this model does not require any delay because experiments had shown that hormone interactions are immediate; however, our proof can be adapted for the delayed case as well. As before, we shall replace the constant parameters by positive $\theta$-periodic functions.

Proposition 1. Assuming that the parameters of (3) are $\theta$-periodic positive continuous functions, then the system has a $\theta$-periodic positive solution.

Proof. If $\mathbf{L} u=\lambda \mathbf{N} u$ with $u=(R, L, T, G)$ and $0<\lambda<1$, then we obtain, for $R\left(t^{*}\right)=R_{\max }$,

$$
d_{0}\left(t^{*}\right) R_{\max } \leq \frac{a_{0}\left(t^{*}\right)+a_{1}\left(t^{*}\right) R_{\max }+a_{2}\left(t^{*}\right) R_{\max }^{2}}{1+c_{1}\left(t^{*}\right) R_{\max }+c_{2}\left(t^{*}\right) R_{\max }^{2}}<C_{R}
$$


for some positive constant $C_{R}$, so we may consider $\mathcal{R}:=\frac{C_{R}}{d_{0_{\min }}}$.

Moreover, if $G$ reaches its maximum value at some $t^{*}$, then from the last equation we obtain:

$$
d_{3}\left(t^{*}\right) G_{\max }<\frac{k_{6}\left(t^{*}\right)}{k_{7}\left(t^{*}\right)}
$$

that is,

$$
G_{\max }<\mathcal{G}:=\left(\frac{k_{6}}{k_{7} d_{3}}\right)_{\max } .
$$

In the same way, we obtain bounds for $L$ from the second equation, denoting again by $t^{*}$ the value in which $L_{\max }$ is achieved:

$$
d_{1}\left(t^{*}\right) L_{\max }<\frac{a_{3}\left(t^{*}\right)+a_{4}\left(t^{*}\right) R\left(t^{*}\right)}{1+c_{3}\left(t^{*}\right) R\left(t^{*}\right)} \leq C_{L},
$$

for some $C_{L}$ depending on the parameters, that is,

$$
L_{\text {max }}<\mathcal{L}:=\frac{C_{L}}{d_{1_{\min }}} .
$$

Regarding $T$, we employ the third equation:

$$
d_{2}\left(t^{*}\right) T_{\max } \leq k_{1}\left(t^{*}\right)+k_{2}\left(t^{*}\right) L\left(t^{*}\right)+\underbrace{\frac{k_{3}\left(t^{*}\right)+k_{4} L\left(t^{*}\right)}{1+k_{5}\left(t^{*}\right) L\left(t^{*}\right)}}_{\leq K_{T}} G\left(t^{*}\right)<k_{1_{\max }}+k_{2_{\max }} \mathcal{L}+K_{T} \mathcal{G}:=C_{T} .
$$

In other words,

$$
T_{\max }<\mathcal{T}:=\frac{C_{T}}{d_{2 \min }}
$$

Lower bounds are obtained similarly. Starting from the second equation, assume that $L$ reaches a minimum at some $t_{*}>0$, then

$$
d_{1}\left(t_{*}\right) L_{\min }>\left(\frac{a_{3}}{1+b_{3} \mathcal{T}+c_{3} \mathcal{R}}\right)_{\min }:=c_{L}
$$

Thus,

$$
L_{\min }>\mathfrak{l}:=\frac{c_{L}}{d_{1_{\max }}}
$$

In the same way, from the third equation we deduce:

$$
T_{\text {min }}>\mathfrak{t}:=\left(\frac{k_{2}}{d_{2}}\right)_{\text {min }} \mathfrak{l}+\left(\frac{k_{1}}{d_{2}}\right)_{\text {min }} .
$$

Next, suppose that $G$ achieves its minimum value at some $t_{*}$, then

$$
d_{3}\left(t_{*}\right) G_{\min }>\frac{k_{6}\left(t_{*}\right) \mathfrak{l}}{1+k_{7}\left(t_{*}\right) \mathcal{L}+q\left(t_{*}\right) \mathcal{R} \mathcal{T}}
$$

which implies

$$
G_{\text {min }}>\mathfrak{g}:=\left(\frac{k_{6} \mathfrak{l}}{d_{3}\left(1+k_{7} \mathcal{L}+q \mathcal{R} \mathcal{T}\right)}\right)_{\text {min }}
$$

Finally, a lower bound for $R$ is obtained from the first equation:

$$
R_{\text {min }}>\left(\frac{a_{0}}{d_{0}\left(1+b_{1} \mathcal{T}+b_{2} \mathcal{T}^{2}+c_{1} \mathcal{R}+c_{2} \mathcal{R}^{2}\right)}\right)_{\text {min }}
$$

Direct computation shows that if $\phi$ and $Q$ are defined as in the preceding section, then

$$
\operatorname{deg}(\phi, Q, 0)= \pm 1
$$

and the continuation theorem applies. 
Models (2) and (3) were proposed to improve a different system which had been considered by Cartwright-Husein [5], according to which, when concentration of LH decays, the rate of testosterone production declines. When concentrations of LH and T decay below a certain level, the production of LHRH from hypothalamus is switched on again. In order to describe this mechanism, the referred paper [5] employed a Heaviside step function for their system,; however, in a later work, Liu and Deng questioned the validity of this model because 'it is hard to give a physical or physiological meaning to the Heaviside step function in the above models, because there are two negative terms in the argument of the step function, we find it hard to imagine that they represent the threshold value of negative feedback.'(see [12]. The model is also discussed and criticized in [10]).

A more realistic situation is presented in the next example.

\section{$3.2 \quad$ A more realistic model}

To describe the population dynamics of testosterone cycle, Greenhalgh-Khan [10] introduced a model based on experimental evidence:

$$
\begin{aligned}
& \frac{d R}{d t}=\frac{b_{1} R(t)}{\left(L(t)+b_{3} T(t)\right)^{k}}-b_{2} R(t), \\
& \frac{d L}{d t}=\frac{c_{1} R(t)^{k} L(t)}{R(t)^{k}+b_{5} T(t)^{k}}-c_{2} L(t), \\
& \frac{d T}{d t}=\left(b_{6} L(t-\tau)-b_{4}\right) T(t) .
\end{aligned}
$$

Here, we shall assume that the parameters of (5) are $\theta$-periodic positive continuous functions.

Proposition 2. Assume that $k, b_{i}$ and $c_{j}$ are strictly positive continuous $\theta$-periodic functions with

$$
c_{1}(t)>c_{2}(t) \quad \text { and } \quad \int_{0}^{\theta} c_{2}(t) d t+\ln \left(\frac{b_{4}}{b_{6}}\right)_{\max }<\frac{1}{k} \ln \left(\frac{b_{1}}{b_{2}}\right)_{\min } .
$$

Then the system admits at least one positive $\theta$-periodic solution.

Proof. In the abstract setting of Theorem 2 observe that if $\mathbf{L} u=\lambda \mathbf{N} u$ for $u:=(R, L, T) \in \mathcal{K}^{\circ}$ and $\lambda \in(0,1)$ then

$$
R^{\prime}(t)>-b_{2}(t) R(t), \quad L^{\prime}(t)>-c_{2}(t) L(t), \quad T^{\prime}(t)>-b_{4}(t) T(t) .
$$

By periodicity, we may fix $t^{*}<t_{*}$ with $t_{*}-t^{*}<\theta$ such that $R\left(t_{*}\right)=R_{\min }$ and $R\left(t^{*}\right)=R_{\max }$. Integrating the inequality $(\ln R)^{\prime}(t)>-b_{2}(t)$ between $t^{*}$ and $t_{*}$ we deduce:

$$
R_{\max }<e^{B_{2}} R_{\min }
$$

where $B_{2}:=\int_{0}^{\theta} b_{2}(t) d t$. Similarly we obtain:

$$
L_{\max }<e^{C_{2}} L_{\min }, \quad T_{\max }<e^{B_{4}} T_{\min }
$$

with

$$
C_{2}:=\int_{0}^{\theta} c_{2}(t) d t, \quad B_{4}:=\int_{0}^{\theta} b_{4}(t) d t .
$$

Moreover, taking a critical point $\xi$ of the function $T$, it is seen from the third equation that

$$
L(\xi-\tau)=\frac{b_{4}}{b_{6}}(\xi)
$$

and hence:

Consequently,

$$
L_{\min } \leq\left(\frac{b_{4}}{b_{6}}\right)_{\max } \text { and } \quad L_{\max } \geq\left(\frac{b_{4}}{b_{6}}\right)_{\min }
$$

and

$$
L_{\max }<e^{C_{2}} L_{\min } \leq e^{C_{2}}\left(\frac{b_{4}}{b_{6}}\right)_{\text {max }}:=\mathcal{L}
$$

$$
L_{\text {min }}>e^{-C_{2}} L_{\text {max }} \geq e^{-C_{2}}\left(\frac{b_{4}}{b_{6}}\right)_{\text {min }}:=\mathfrak{l}
$$


In the same way, observe from the first equation that, for some value (which we call again $\xi$ ),

$$
\left(\frac{b_{1}(\xi)}{b_{2}(\xi)}\right)^{1 / k}=L(\xi)+b_{3}(\xi) T(\xi)
$$

Thus we deduce:

$$
\begin{gathered}
T_{\text {max }}<e^{B_{4}} T_{\min } \leq e^{B_{4}}\left(\frac{\left(\frac{b_{1}}{b_{2}}\right)^{1 / k}-\mathfrak{l}}{b_{3}}\right)_{\max }:=\mathcal{T} \\
T_{\min }>e^{-B_{4}} T_{\max } \geq e^{-B_{4}}\left(\frac{\left(\frac{b_{1}}{b_{2}}\right)^{1 / k}-\mathcal{L}}{b_{3}}\right)_{\text {min }}:=\mathfrak{t}
\end{gathered}
$$

We remark that $\mathfrak{t}>0$, because $\frac{b_{1}}{b_{2}}(t)>\mathcal{L}^{k}$ for all $t$. Finally, take the second equation and observe as before that, for some $\xi$,

that is

$$
\frac{c_{1}(\xi) R(\xi)^{k}}{R(\xi)^{k}+b_{5}(\xi) T(\xi)^{k}}=c_{2}(\xi)
$$

Since $c_{2}(t)<c_{1}(t)$ for all $t$, it follows that

$$
\left(c_{1}(\xi)-c_{2}(\xi)\right) R(\xi)^{k}=c_{2}(\xi) b_{5}(\xi) T(\xi)^{k}
$$

$$
\begin{gathered}
R_{\text {max }}<e^{B_{2}} R_{\text {min }} \leq e^{B_{2}}\left(\frac{c_{2} b_{5}}{c_{1}-c_{2}}\right)_{\text {max }}^{1 / k} \mathcal{T}:=\mathcal{R}, \\
R_{\text {min }}>e^{-B_{2}} R_{\text {max }} \geq e^{-B_{2}}\left(\frac{c_{2} b_{5}}{c_{1}-c_{2}}\right)_{\text {min }}^{1 / k} \mathfrak{t}:=\mathfrak{r}>0 .
\end{gathered}
$$

In order to complete the proof, let us proceed as we did in the proof of our main theorem. Define $\phi$ as before over the set

It is directly verified that

$$
\bar{Q}:=\left\{(R, L, T) \in \mathbb{R}^{3}: \mathfrak{r} \leq R \leq \mathcal{R}, \mathfrak{l} \leq L \leq \mathcal{L}, \mathfrak{t} \leq T \leq \mathcal{T}\right\}
$$

$$
\phi_{3}(R, \mathcal{L}, T)=\frac{1}{\theta} \int_{0}^{\theta} T\left(b_{6}(t) \mathcal{L}-b_{4}(t)\right) d t=T\left(\overline{b_{6}} e^{C_{2}}\left(\frac{b_{4}}{b_{6}}\right)_{\text {max }}-\overline{b_{4}}\right)>0
$$

because, in virtue of Cauchy's mean value theorem, there exists $\xi$ such that $\frac{\bar{b}_{4}}{\bar{b}_{6}}=\frac{b_{4}(\xi)}{b_{6}(\xi)} \leq\left(\frac{b_{4}}{b_{6}}\right)$ max . In the same way, it is deduced that

Next, we verify that

$$
\phi_{3}(R, \mathfrak{l}, T)=\frac{1}{\theta} \int_{0}^{\theta} T\left(b_{6}(t) \mathfrak{l}-b_{4}(t)\right) d t=T\left(\overline{b_{6}} e^{-C_{2}}\left(\frac{b_{4}}{b_{6}}\right)_{\min }-\overline{b_{4}}\right)<0 .
$$

$$
\phi_{1}(R, L, \mathcal{T})=\frac{R}{\theta} \int_{0}^{\theta}\left(\frac{b_{1}(t)}{\left(L+b_{3}(t) \mathcal{T}\right)^{k}}-b_{2}(t)\right) d t<\frac{R}{\theta} \int_{0}^{\theta}\left(\frac{b_{1}(t)}{\left(\mathfrak{l}+b_{3}(t) \mathcal{T}\right)^{k}}-b_{2}(t)\right) d t
$$

thus, enlarging $\mathcal{T}$ if necessary we conclude that $\phi_{1}(R, L, \mathcal{T})<0$. On the other hand, since $\frac{b_{1}}{b_{2}}(t)>\mathcal{L}^{k}$ we deduce, by letting $\mathfrak{t}$ be smaller if necessary, that

$$
\phi_{1}(R, L, \mathfrak{t})>\frac{R}{\theta} \int_{0}^{\theta}\left(\frac{b_{1}(t)}{\left(\mathcal{L}+b_{3}(t) \mathfrak{t}\right)^{k}}-b_{2}(t)\right) d t>0 .
$$

Finally, let us analyse the second coordinate for $(R, L, T) \in \bar{Q}$

$$
\phi_{2}(R, L, T)=\frac{L}{\theta} \int_{0}^{\theta} \frac{\left(c_{1}(t)-c_{2}(t)\right) R^{k}-c_{2}(t) b_{5}(t) T^{k}}{R^{k}+b_{5}(t) T^{k}} d t .
$$

Since the bounds for $T$ are already fixed and $c_{1}(t)>c_{2}(t)$ for all $t$, it is seen that

$$
\phi(R, L, T)>0 \quad \text { for } R \gg 0
$$

and

$$
\phi(R, L, T)<0 \quad \text { for } R \ll 1 .
$$

This means that we may make $\mathcal{R}$ larger and $\mathfrak{r}$ smaller if necessary, and the proof follows as in Theorem 1. 


\section{Summary and Discussion}

Topological degree was used for proving existence of stable equilibrium in a generic model of glandular cycle. This theory allowed to demonstrate the existence of positive periodic solutions when parameters are replaced by fixed periodic functions. It is observed that the results are also valid in the absence of delays; however, the study of delayed systems is of great interest because as shown in [7], in many cases delayed models fit experimental data better, particularly when feedback is present.

The relevance of finding periodic solutions in biological models like the ones studied in this work relies mainly on the fact that periodic functions represent natural cycles, such as hormonal processes.

In the literature, there exist many works that study the cycle of testosterone with different models based on experimental data. We show that topological degree can be successfully applied to find positive periodic orbits for some of these models in the non-autonomous case. It is worthy mentioning that, for diverse biological cycles, the behaviour is characterized by models with periodic parameters; thus, the present paper provides a useful mathematical tool to understand such models.

As mentioned, the existence of $\theta$-periodic solutions in this paper does not depend on the delay but only on the $\theta$ periodicity of the parameters. When the parameters are constant, the fact that the degree is equal to \pm 1 implies the existence of a positive equilibrium which, in particular, is a solution of the problem in the space $C_{\theta}$. Thus the method, at this stage, does not give any clue about the existence of (non-constant) periodic solutions. It would be interesting to prove the existence of such periodic solutions for the autonomous case and investigate the role of the delay. At first sight topological degree methods might still be effective for these situations, so this might be the topic of future research. Also, it would be interesting to consider a more general situation, in which the parameters are not periodic but almost-periodic functions, which attracted the attention of many researchers in the last decades. Here, the topological degree cannot be used anymore because of the lack of compactness of the associated operator; thus, a different approach is required, such as the use of fixed points in cones under monotonicity conditions that avoid the compactness assumption.

\section{Acknowledgements}

The authors thank to the anonymous reviewers for the careful reading of the manuscript and their thoughtful comments. This work has been partially supported by projects CONICET PIP 11220130100006 CO and UBACyT 20020160100002BA.

\section{References}

[1] P. Amster And L. Idels, Existence theorems for some abstract nonlinear non-autonomous systems with delays, Commun. Nonlinear Sci. Numer. Simulat. 19 (2014) 2974-2982.

[2] P. Amster And L. Idels, Periodic solutions in general scalar non-autonomous models with delays, Nonlinear Differential Equations and Applications NoDEA, 1577-1596, Vol 20 (2013).

[3] D. Blache, S. Zhang, G. Martin, Fertility in male sheep: modulators of the acute effects of nutrition on the reproductive axis of male sheep, Reprod Suppl. 2003; 61:823-829.

[4] R. Caminos-Torres, L. Ma, P. J. Snyder, Testosterone-Induced Inhibition of the LH and FSH Responses to Gonadotropin-Releasing Hormone occurs slowly, Journal of Clinical Endocrinology and Metabolism, Vol 44, No6 (1977), 1142-1153.

[5] M. Cartwright, M. Husain, A Model for the Control of Testosterone Secretion, J. theor. Biol, Vol 123 (1986), 239-250.

[6] P. DAs, A. B. Roy AND A. DAs, Stability and oscillations of a negative feedback delay model for the control of testosterone secretion, BioSystems 32 (1994) 61-69.

[7] T. Ferasyi, R. Barrett, D. Blache, and G. Martin, Modeling the Male Reproductive Endocrine Axis: Potential Role for a Delay Mechanism in the Inhibitory Action of Gonadal Steroids on GnRH Pulse Frequency, Endocrinology, 157: 2080-2092 (2016)

[8] A. Goldbeter, Biochemical Oscillations and Cellular Rhythms, Cambridge University Press (1996).

[9] B. Goodwin, Oscillatory Behaviour in Enzymatic Control Processes, Adv. Enzyme Regul. 3 (1965), 425-438.

[10] D. Greenhalgh, Q. J. A. Khan, A Delay Differential Equation Mathematical Model for the control of the hormonal system of the hypothalamus, the pituitary and the testis in man. Nonlinear Analysis: Theory, Methods and Applications, 71 No. 12 (2009), 925-935. 
[11] S. Hastings, J. Tyson And D. Webster, Existence of Periodic Solutions for Negative Feedback Cellular Control Systems, Journal of Differential Equations 25 (1976), 39-64.

[12] B. Liu, G. Deng, An Improved Mathematical Model of Hormone Secretion in the Hypothalamo-Pituitary-Gonadal Axis in Man, J. theor. Biol, Vol 150 (1991), 51-58.

[13] J. Murray, Mathematical Biology. I. An Introduction, Springer, New York 2001.

[14] S. RUAN AND J. WeI, On the zeros of a third degree exponential polynomial with applications to a delayed model for the control of testosterone secretion, IMA Journal of Math. Applied in Medicine and Biology 18 (2001), 41-52.

[15] W. SмIтh, Hypothalamic regulation of pituitary secretion of luteinizing hormone. II. Feedback control of gonadotropin secretion, Bulletin of Mathematical Biology, Vol. 42 (1980), 57-78. 